\title{
UFG-Microstructures by Linear Flow Splitting
}

\section{Clemens Müller ${ }^{1, a}$, Tilman Bohn ${ }^{1, b}$, Enrico Bruder ${ }^{1, c}$ and Peter Groche $e^{2, d}$}

${ }^{1}$ Division Physical Metallurgy, Technische Universität Darmstadt, Petersenstrasse 23, 64287 Darmstadt, Germany

\author{
${ }^{2}$ Institute for Production Engineering and Forming Machines, Technische Universität Darmstadt, \\ Petersenstraße 30, 64287 Darmstadt, Germany \\ ac.mueller@phm.tu-darmstadt.de, bt.bohn@phm.tu-darmstadt.de, \\ ce.bruder@phm.tu-darmstadt.de,groche@ptu.tu-darmstadt.de
}

Keywords: Linear flow splitting, Severe deformation, Ultrafine grains, Hardness, HSLA steel

\begin{abstract}
Linear flow splitting is a new continuous cold forming process where the edge of a sheet metal is formed into two flanges by splitting and supporting rolls. Thus the production of bifurcated profiles from sheet metal without lamination of material becomes feasible. The production of such structures takes place incrementally in a modified roll forming machine. Experimental investigateons on a HSLA steel show, that even at a surface increase of the sheet edge of about $1800 \%$ no cracks were nucleated in the profiles. EBSD measurements in the splitting centre reveal that similar to other SPD processes UFG microstructures develop in the processing zone. Thus a steady state is reached in the processing zone where increasing strain has no more (or little) influence on the materials properties i.e. its deformability, as it is typical for SPD-processes. The formation of UFG microstructures is considered to be a mandatory condition for the linear flow splitting process, as it improves the formability of the material to the extremely high level required for this process. The mechanical properties of profiles produced by linear flow splitting are characterised by large gradients, depending on the local deformation and the resulting microstructure. Very high hardness is measured at the former processing zone, i.e. the splitting centre and the flange surface, where severe plastic deformation takes place and UFG microstructures are present. In direction to lower deformation i.e. with increasing distance to the splitting ground or flange surface the hardness decreases close to the level of the undeformed material. In the present paper the linear flow splitting process is introduced and the microstructural development in the process zone is discussed on the base of EBSD measurements on profiles of the steel ZStE 500. The repartition of mechanical properties in a bifurcated profile is demonstrated by detailed hardness measurements.
\end{abstract}

\section{Introduction}

The global competition and the limited material resources force companies to increase their product functionality as well as to optimize the manufacturing cycles. This involves an increased complexity of the products and simultaneously enforces improvements of the manufacturing technologies. For cold forming processes materials would be used whose high performances do not limit the production process by exhausted formability. Therefore UltraFine Grained (UFG) microstructures with grain dimensions in the submicron range, characterized by high mechanical strength as well as high ductility, are of great technological and scientific interest [1]. Presently UFG materials are manufactured by several methods of Severe Plastic Deformation (SPD), like Equal Channel Angular Pressing (ECAP) [2], Accumulative Roll Bonding (ARB) [3] and High Pressure Torsion (HPT) [4]. All these SPD methods are characterized by high hydrostatic compression stresses, which enable extremely high deformations in the work piece. Cells, cellblocks and microbands are formed within the grains and a subgrain structure of Low Angle Grain Boundaries (LAGBs) is created. With further deformation the LAGBs develop to High Angle Grain Boundaries (HAGBs) and lead to a grain refinement with grain sizes in the submicron range [5,6]. The typical strains to generate homogeneous UFG microstructures with a majority fraction of HAGBs is about $\varepsilon=4-8$ $[3,5,7]$, depending on the method and the material, respectively. Originally, these processes were developed for fundamental investigations $[1,8,9$,$] , but due to their excellent properties an interest$ 
for the industrial utilization of UFG metals processed by SPD evolved, especially for aerospace and medical applications. For industrial applications one major inconvenience of these SPD processes is that most of them are not (or difficultly) extendable to continuous processes. The new linear flow splitting technology is a continuous process where UFG microstructures can be achieved in real components.

\section{Principles of linear flow splitting}

During linear flow splitting a plane sheet metal is transformed by a specific tooling system at ambient temperature [10]. The edge of a sheet metal (sheet or coil) is formed into two flanges by splitting and supporting rolls. The continuous production takes place in a modified roll forming machine, as it is shown in Fig. 1. The tool system is fixed and it consists of obtuse angled splitting rolls and supporting rolls. Fig. 2 shows the forming of one band edge into two flanges. The work piece is moved with help of the supporting rolls. The forming process is carried out by the splitting roll. The splitting roll drives into the band edge by an incremental splitting depth $y_{\text {inc. The }}$ continuous production takes place gradually from stage to stage and the total splitting depth $\mathrm{y}_{\text {tot }}$ results from the sum of the incremental splitting depths $y_{\text {inc,i }}$ of the individual stages. The produced bifurcated profile is marked by a web and two flanges. The surface beneath the splitting roll was originally the band edge.
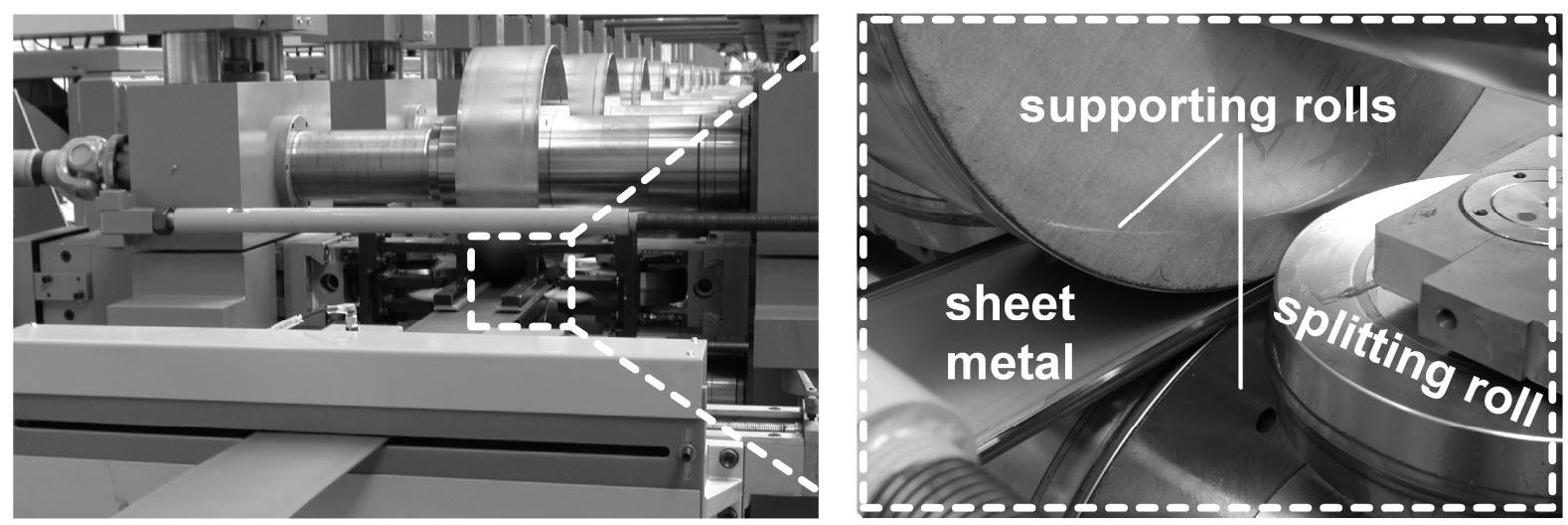

Fig. 1: Modified roll forming machine for the continuous production of bifurcated profiles

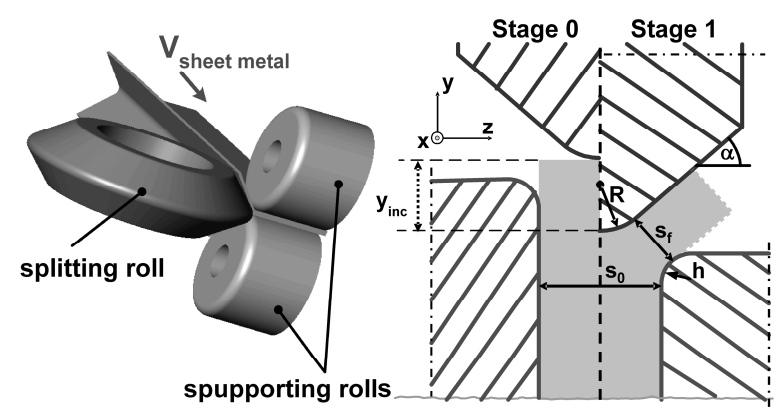

Fig. 2: Process principle (left hand side) and geometrical characteristics of linear flow splitting (right hand side)

As Fig. 3 shows, the surface of the band edge increases during the forming process. Considering a constant volume and with the assumption of a plain-strain state, the flange length and therefore also the elongation can be determined [11]. A linear correlation between the averaged theoretically determinable strain and the splitting depth can be calculated. Experimental investigations have shown that for the configuration - angle of the splitting roll $\alpha=30^{\circ}$; working radius of the splitting roll $\mathrm{R}=5 \mathrm{~mm}$; working radius of the supporting roll $\mathrm{h}=5 \mathrm{~mm}$; sheet metal thickness $\mathrm{s}_{0}=6 \mathrm{~mm}$; flange thickness $\mathrm{s}_{\mathrm{f}}=3,4 \mathrm{~mm}-\mathrm{a}$ theoretical strain $\varepsilon_{\text {theor }}$ from 16.01 after a splitting depth of $55 \mathrm{~mm}$ was acquired. That corresponds to a surface increase of the band edge up to $1800 \%$ [12]. 


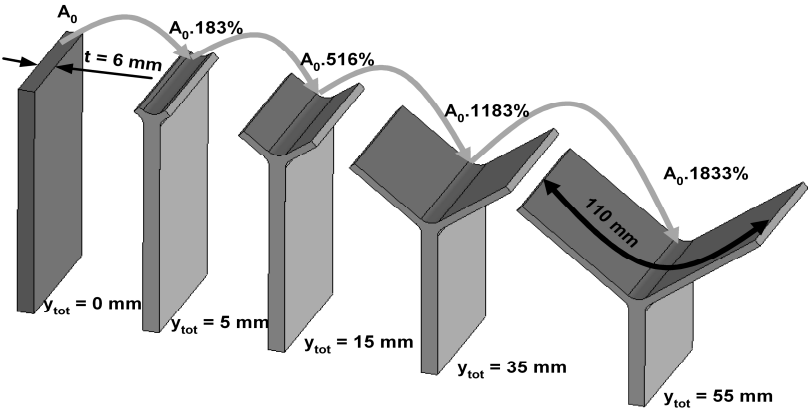

Fig. 3: Surface increase by linear flow splitting [12]

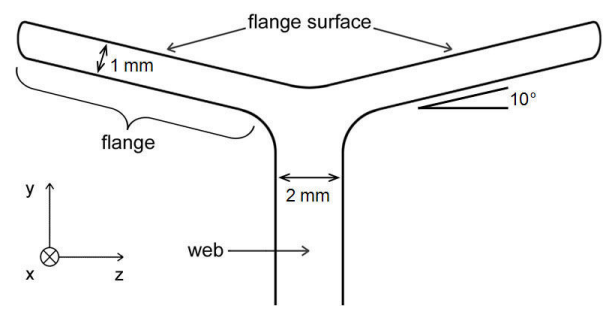

Fig. 4: Profile scheme

These large strains indicate that other mechanisms of plastic deformation occur, which counteract the effects of work hardening and thus increase the formability. It is obvious that profiles produced by linear flow splitting are characterised by strongly inhomogeneous deformation resulting in microstructural and property gradients. The present paper gives more insight in the specific deformation process and highlights the resulting microstructure as well as local properties of profiles produced by linear flow splitting.

\section{Experimental}

The material used in this investigation is a HSLA steel sheet of the grade ZStE $500(0.07 \mathrm{wt} \% \mathrm{C}$, $0.71 \mathrm{wt} \% \mathrm{Mn}, 0.1 \mathrm{wt} \% \mathrm{Cr}, 0.047 \mathrm{wt} \% \mathrm{Si}, 0.034 \mathrm{wt} \% \mathrm{Nb}$ and $0.016 \mathrm{wt} \% \mathrm{Al}$ ) with a thickness of $2 \mathrm{~mm}$, designed for deep drawing. Linear flow splitting was conducted at the Institute for Production Engineering and Forming Machines (Technische Universität Darmstadt) to a total splitting depth ( $\left.\mathrm{y}_{\text {tot }}\right)$ of $20 \mathrm{~mm}$ with an incremental splitting depth $\left(\mathrm{y}_{\text {inc }}\right)$ of $1 \mathrm{~mm}$, a flange thickness $\left(\mathrm{s}_{\mathrm{f}}\right)$ of $\sim 1 \mathrm{~mm}$ and an angle of the flanges $(\alpha)$ of $10^{\circ}$. Shape and dimensions of the resulting profiles are shown in Fig. 4.

Metallographic specimens were prepared from the cross section of the profiles. For EBSD (Electron BackScatter Diffraction) measurements the specimens were vibropolished for at least 8 hours on a $50 \mathrm{~nm}$ mixed oxide suspension. EBSD measurements were carried out using a FEG SEM fitted with a TSL EBSD system. Low angle grain boundaries have been defined between $2^{\circ}$ and $15^{\circ}$ misorientation and high angle grain boundaries above a misorientation of $15^{\circ}$.

Hardness measurements (HV0.05) were performed on the cross section of the profile from the splitting center to the web and parallel to the flange surface.

\section{Results}

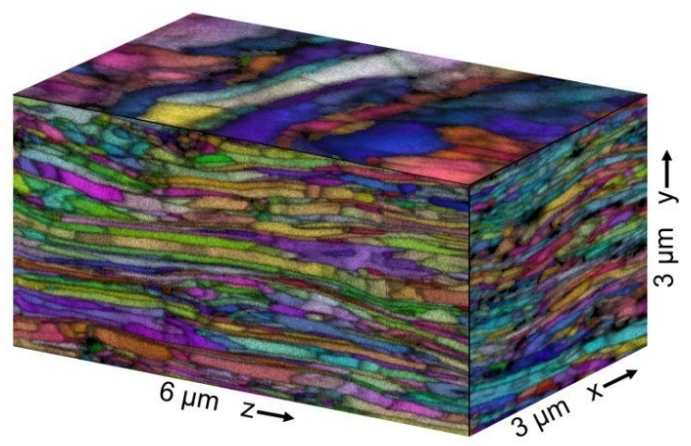

Fig. 5: EBSD maps (image quality + inverse pole figure) of the planes perpendicular to the $\mathrm{x}-, \mathrm{y}$ - and $\mathrm{z}$-axis $50 \mu \mathrm{m}$ underneath the flange surface 

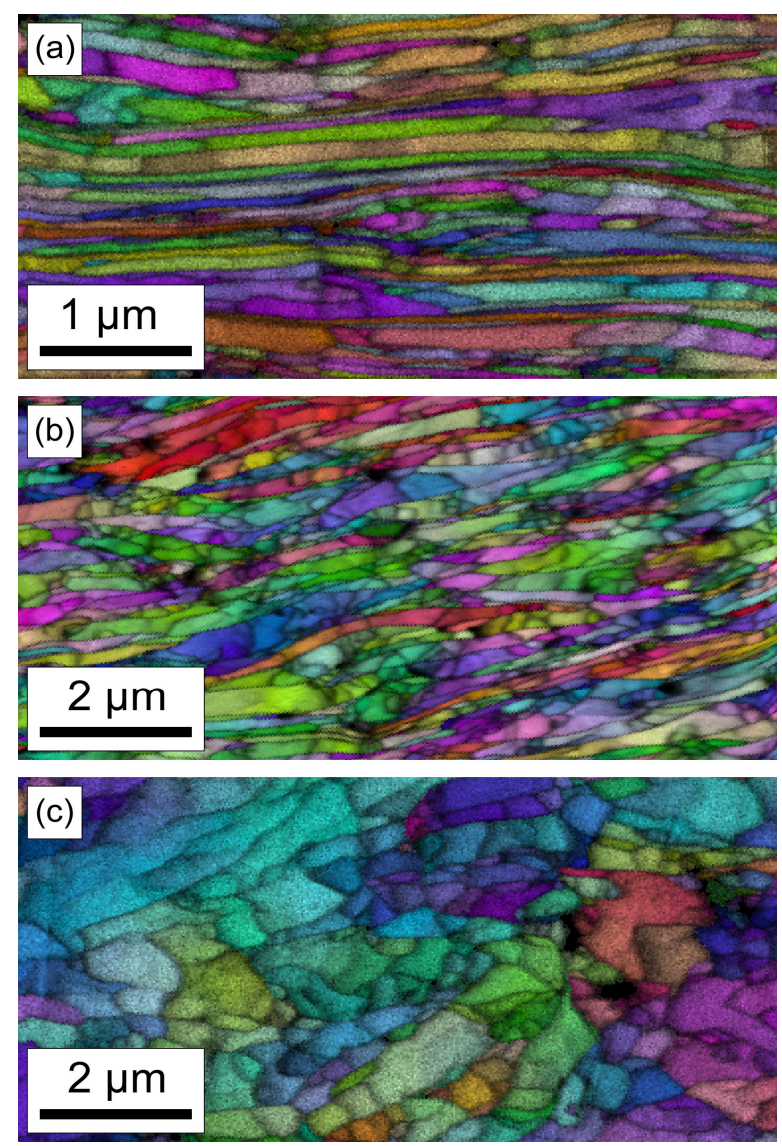

Fig. 6: EBSD maps (image quality + inverse pole figure) at different distances to the flange surface: (a) $50 \mu \mathrm{m}$, (b) $250 \mu \mathrm{m}$, (c) $550 \mu \mathrm{m}$.

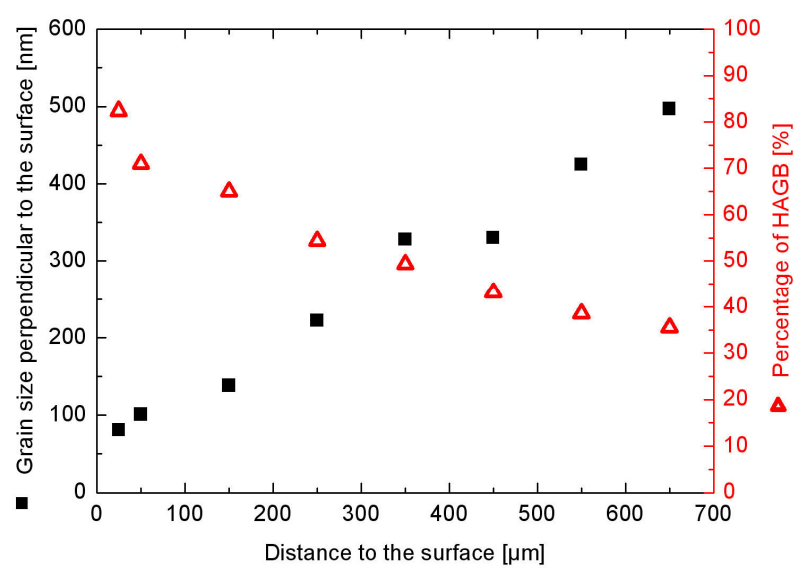

Fig. 7: Grain dimensions perpendicular to the surface and HAGB fraction vs. distance to the surface.

Microstructure: The microstructure of the as received HSLA steel consists of equiaxed ferrite grains with small cementite precipitations located at the grain boundaries. The average grain size of the sheet material measured by optical microscopy was $\sim 6 \mu \mathrm{m}$. After the rolling process the microstructure in the highly deformed regions, i.e. the splitting center and the flange surface, cannot be resolved by optical microscopy anymore. EBSD measurements reveal that linear flow splitting leads to an UFG microstructure in the splitting center and accordingly the flange surface area. Due to the preferential flow direction of the material (perpendicular to the splitting direction) a pancake like UFG micro-structure develops, which is exemplarily shown in Fig. 5 for a position $50 \mu \mathrm{m}$ underneath the flange surface. The maximum grain dimensions are parallel to the z-axis and the minimum parallel to the y-axis. The following micrographs concentrate on the $\mathrm{y} \times \mathrm{z}$ plane as it includes these two directions. Note that although the structure in the $\mathrm{x} \times \mathrm{z}$ plane is comparatively coarse its area-related average grain size is still less than $1 \mu \mathrm{m}$. Detailed investigations on the microstructure of the flanges reveal a grain size gradient perpendicular to the flange surface which is presented in Fig. 6 by EBDS maps (combined image quality + inverse pole figure). The elongated grains near the surface are mostly separated by HAGBs $(80 \%)$. Within $50 \mu \mathrm{m}$ to the flange surface the average grain dimensions along the y-axis (line intersection perpendicular to the split surface) are below $100 \mathrm{~nm}$. With increasing depth, i.e. distance to the split surface, the grain structure becomes coarser and more equiaxed. This coarsening is associated with a decreasing fraction of HAGBs from over $80 \%$ close to the flange surface to about $30 \%$ at the lower side of the flange (Fig. 7). No microstructural gradients are observed parallel to the flanges except at the flange tip, about $4 \mathrm{~mm}$ in length. Thus the UFG microstructures shown in Fig. 6 are representative for a large part of the flanges.

Hardness measurements: The average hardness of the as received material was measured to 196 HV0.05 and is homogeneous across the cross section. Hardness measurements on cross sections of flanges from the splitting center to the flange tips $(25 \mu \mathrm{m}$ below the surface) reveal a constant hardness of $365 \mathrm{HV} 0.05$ parallel to the surface over a wide range of the flange in spite of the continuously increasing deformation towards the flange centre (Fig. 8). This zone of constant hardness correlates well with the presence of UFG microstructure mentioned above. Only at the flange tips, i.e. the surface created at the first splitting steps, the expected correlation - increasing hardness with increasing deformation - can be observed. 
In direction perpendicular to the flange surface the split profiles show a steep hardness gradient that is shown in Fig. 9 for measurements at the flange center towards the web of the profile. The highest hardness is measured at the surface with a value of $365 \mathrm{HV} 0.05$. With increasing distance to the surface the hardness decreases and reaches a constant level in a depth of $1 \mathrm{~mm}$ of $225 \mathrm{HV} 0.05$, which is about $30 \mathrm{HV} 0.05$ higher than the as received material. The same behavior (identical hardness distribution) was found for the whole zone of constant hardness in Fig. 8.

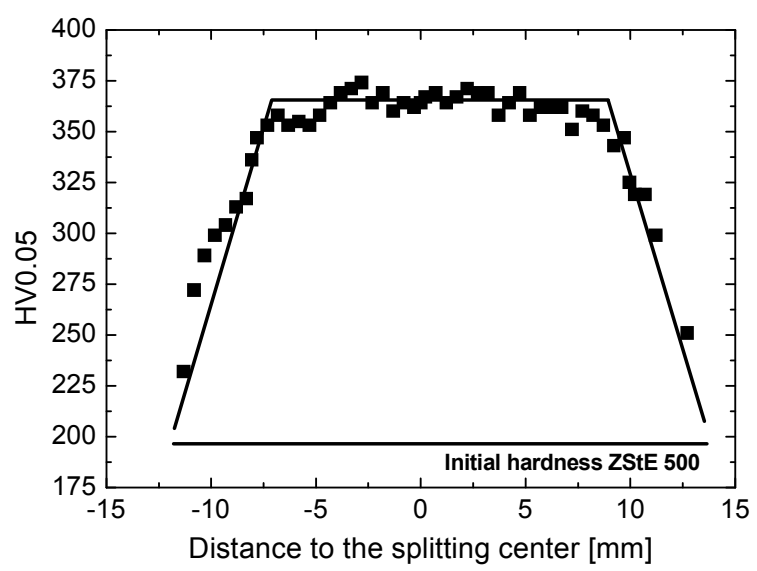

Fig. 8: Hardness repartition parallel to the flange surface $(25 \mu \mathrm{m}$ underneath the surface)

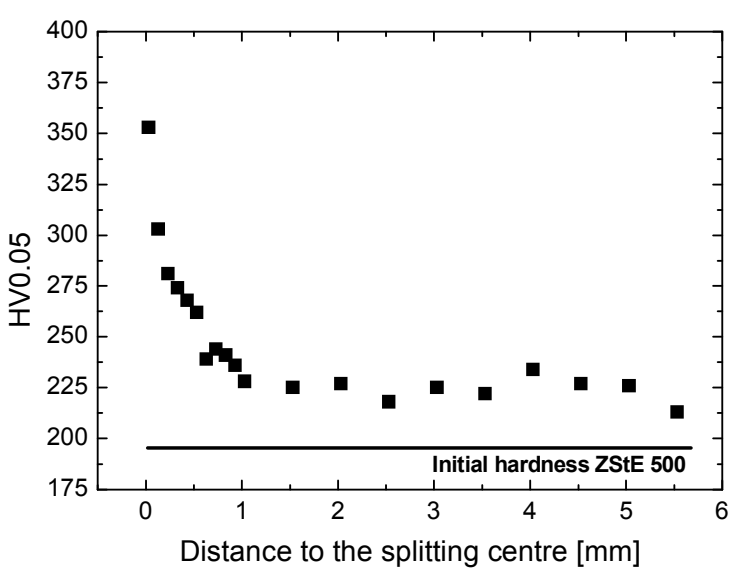

Fig. 9: Hardness repartition from the splitting center to the web

\section{Discussion}

Complex deformation and flow processes of the material are characteristic for linear flow splitting. To get a better insight into these processes it is advantageous to separate the split profile in two zones: The process zone where the deformation takes place and the flange zone where no further deformation takes place. The process zone is roughly limited by the area within a triangle between the contact points of the splitting and the supporting rolls with the sheet material (see Fig. 2 right hand side). Similar to other SPD processes linear flow splitting generates high hydrostatic compressive stresses in the process zone. These compressive stresses obviously increase the formability of the material to a degree that UFG microstructures can develop. Due to the deformation gradient beneath the splitting roll an UFG gradient microstructure develops where the grain size increases with increasing distance to the splitting roll, i.e. the split surface. In the flange zone where no further deformation takes place the microstructural evolution of previous splitting steps, i.e. of previous processing zones is present. Therefore it is not surprising to find similarities to the observations mentioned above for the process zone: UFG microstructures in the surface area with a grain size gradient perpendicular to the flange surface. This microstructural gradient is combined with a hardness gradient due to the changing grain size. However the constant hardness parallel to the surface over a wide range of the flange (Fig. 8) is surprising at first glance, as FEM modeling clearly indicates an increasing deformation degree towards the splitting center $[10,12]$. By the microstructural evolution i.e. the formation of UFG microstructures in the process zone this effect becomes plausible. As it is well known for SPD processes like ECAP or HPT, UFG microstructures and mechanical properties become almost independent of further deformation when a certain strain is exceeded $[4,7,13]$. In the HSLA steel used for the present investigations this strain is reached at a flange length of about $4 \mathrm{~mm}$ where the zone of constant hardness (and constant grain size) parallel to the flange surface starts. This finding implies the evolution of a steady state in the processing zone of the linear flow splitting process. This assumption is supported by measurements of the rolling forces that remain almost constant when a flange length of $4 \mathrm{~mm}$ is reached [11]. The steady state in the process zone (that can only be achieved by the formation of UFG microstructures) has three important consequences for the linear flow splitting process:

1. Microstructural and property gradients are limited to the direction perpendicular to the flange surface (except the flange tips). 
2. No limitation of the splitting due to exhausted formability is expected. Reaching this steady state, i.e. the formation of UFG microstructures is even a mandatory condition for the linear flow splitting process if large flange dimensions are requested.

3. The splitting process is not limited by increasing rolling forces, i.e. the strength and elastic deformation of the rolling tools.

Due to the formation of UFG microstructures the flanges show a very high hardness on the surface that makes the profiles especially interesting for applications where high wear resistance and good fatigue properties are required. Furthermore excellent tensile properties, i.e. high yield strength combined with acceptable ductility can be expected for the profiles due to the presence of UFG microstructures in the flanges.

\section{Summary and conclusions}

The HSLA steel ZStE 500 was used to investigate the microstructure and mechanical properties of bifurcated profiles produced by linear flow splitting.

Linear flow splitting leads to the formation of UFG microstructures at the surface area of the split flanges. Grain size gradients perpendicular to the flange surface are characteristic for profiles produced by linear flow splitting.

Due to the formation of UFG microstructures a steady state is reached in the process zone, where increasing deformation (i.e. splitting depth) has no more or little influence on microstructure and resulting properties. Thus work hardening and the related reduction of formability are no limiting parameters for the splitting process.

The flanges exhibit very high hardness on the split surface. This makes the profiles especially interesting for applications where high wear resistance and good fatigue properties are required.

\section{Acknowledgements}

The investigations presented in this paper are supported by the German Research Foundation (DFG). The authors thank the DFG for funding the subproject $\mathrm{C} 1$ of the Collaborative Research Center 666 "Integral sheet metal design with higher order bifurcations - Development, Production, Evaluation".

\section{References}

[1] R.Z. Valiev, Y. Estrin, Z. Horita, T.G. Langdon, M.J. Zehetbauer, Y.T. Zhu: JOM 58 (2006), p. 33

[2] M. Furukawa, Z. Horita, M. Nemoto and T.G. Langdon: J. Mater. Sci. Vol 36 (2001), p. 2835

[3] N. Tsuji N, Y. Saito, Y. Utsunomiya, S. Tanigawa: Scripta Mater. 40 (1999), p. 795

[4] A.P. Zhilyaev, G.V. Nurislamova, B.-K. Kim, M.D. Baró, J.A. Szpunar and T.G. Langdon: Acta Mater. Vol. 51 (2003), p. 753

[5] Y. Iwahashi, Z. Horita, M. Nemoto, T.G. Langdon: Acta Mater. 45 (1997), p. 4733

[6] D.A. Hughes, N. Hansen: Acta Mater. 45 (1997), p. 3871

[7] P.B. Pragnell, J.R. Bowen, A. Gholinia, in: Proc. of the 22nd Risø Int. Symp. on Materials Science, edited by A.R. Dinesen, M. Eldrup, D. Juul Jensen, S. Linderoth, T.B. Pederson, N.H. Pryds, A. Schrøder Pedersen, J.A. Wert, Risø National Laboratory, Roskilde, Denmark, 2001

[8] Y.T. Zhu, T.C. Lowe, T.G. Langdon: Scripta Mater. 51 (2004), p. 825

[9] R.Z. Valiev, R.K. Islamgaliev, I.V. Alexandrov: Prog. Mater. Sci. 45 (2000), p. 103

[10]P. Groche, J. Ringler, D. Vucic: Key Engineering Materials 344 (2007), p. 251

[11]P. Groche, D. Vucic, M. Jöckel: Journal of Materials Processing Technology 183 (2007), p. 249

[12]C. Müller, T. Bohn, E. Bruder, T. Bruder, V. Landersheim, C. el Dsoki, P. Groche, D. Veleva: Mat.-wiss. u. Werkstofftech. 38 (2007), p. 842

[13]H.S. Kim, W.S. Ryu, M. Janecek, S.C. Baik, Y. Estrin: Adv. Eng. Mater. 7 (2005), p. 43 